\title{
Smooth Variable Structure Filter for Pneumatic System Identification
}

\author{
Mohammad A. Al-Shabi \\ Department of Mechatronics Engineering \\ Philadelphia University \\ Amman, Jordan \\ maqas2002@yahoo.com
}

\author{
Ashraf Saleem \\ Department of Mechatronics Engineering \\ Philadelphia University \\ Amman, Jordan \\ asaleem@philadelphia.edu.jo
}

\author{
Tarek A. Tutunji \\ Department of Mechatronics Engineering \\ Philadelphia University \\ Amman, Jordan \\ ttutunji@yahoo.com
}

\begin{abstract}
The Smooth Variable Structure Filter (SVSF) is a newly-developed predictor-corrector filter for state and parameter estimation [1]. The SVSF is based on the Sliding Mode Control concept. It defines a hyperplane in terms of the state trajectory and then applies a discontinuous corrective action that forces the estimate to go back and forth across that hyperplane. The SVSF is suitable for fault detection and identification applications because of its stability and robustness in modeling uncertainties. The SVSF has two indicators of performance; the a posteriori output error and the chattering. The latter -as a signalcontains the system's information which is proven and explored in this paper. The SVSF is applied for the identification of pneumatic systems in order to verify the proposed method. Furthermore, the proposed method is compared with neural network and the results reveal that SVSF is better in identifying nonlinear systems.
\end{abstract}

Keywords- SVSF, System Identification, Pneumatic Systems.

\section{INTRODUCTION}

"From earliest time, people have been concerned with interpreting observations and making estimates and predictions." Kailath said in [2] and continued by "Neugebauer has noted that the Babylonians used a rudimentary form of Fourier series for such purposes". The beginning of the "theory of estimation" can be tracked back to 1632, when Galileo tried to minimize the error of some functions [2]. In 1795 , Gauss introduced and used the method of least squares to locate the asteroid Ceres, although Legendre first published it independently in $1805[2,3]$. These were followed by numerous investigations and studies pertaining to the least squares method, leading to the pioneering work of Wiener in the 1940's [2,4,5]. In 1942, Wiener gave the first explicit solutions for the problem of estimating a stochastic process using the least squares method, referred to as the Wiener Filter $[2,5,6]$. In [2], Kailath gave a brief history of the estimation problem focusing on the period from 1930s to 1960s. In that article, he summarized the history of optimal filters up to the Kalman Filter (KF) which was one of the earliest filters that was implemented in a predictor-corrector form.

The history of estimation was continued in [4] focusing on the period from 1960s to 2000s. He specifically considered the non-linear Bayesian filtering problems that include the Particle Filter. The Bayesian approach was applied to estimation in stochastic processes in 1964 by Ho and Lee [4,7]. They introduced the iterative Bayesian filtering and explored the concepts of "the sequential state estimation problem" [4]. Later in the 1980's and the 1990's, the Bayesian filtering was expanded to include the state space structure. During the twentieth century, the "optimality" derivatives were formulated specifically in the Bayesian framework. The notion of optimality relies on minimizing a measure referred to as the cost function $[4,8,9]$.

Simultaneously with the development of the optimal filters, another type of filter started to rise and take place in estimation utilizing the principles of the Variable Structure System and Control as well as the Sliding Mode Control. These filters and observers are referred to as Sliding Mode Observers (SMO). These observers are used widely in fault detection and signal reconstruction problems due to their robustness and stability against uncertainties. An early example of the SMO was done by Slotine in 1986 and 1987. In [10,11], Slotine et al. modified the Luenberger Observer by adding a discontinuous element that tolerates the nonlinearity in the system. They described their observer using the SMC mold and explored the effectiveness of its gains mathematically. At the same period, Walcott et al. published a landmark paper [12] on the linear SMO algorithm and its design methodologies. Later in 1994, Edwards et al. expanded the design algorithm to a more general form using symbolic manipulation and defined an explicit design algorithm, $[13,14]$. At the beginning of the $21^{\text {st }}$ century, Tan and Edwards presented their observer as an extended version of the Walcott and Zak observer, with less constraint and a simpler design method [15].

In 2003, a new estimation method referred to as the Variable Structure Filter (VSF) was proposed for its applications to linear systems. This method is a version of the SMO formulated in a recursive predictor-corrector form $[16,17,18]$. In 2006, the VSF was revised to a new form, referred to as the Extended Variable Structure Filter (EVSF), to accommodate nonlinear systems. The EVSF uses the linearized system and output matrices to obtain its gain [19]. Later in 2007, a revised version of the VSF, referred to as the Smooth Variable Structure Filter (SVSF), was proposed for its applications to linear and nonlinear systems [1]. The benefits of the SVSF over the VSF and the EVSF are its simplicity and the absence of linearizing the system matrix.

The SVSF is discussed in further detail in section (II). This includes the filter's structure and its concepts. The chattering is developed mathematically in section (III). The new method used to obtain the system information from chattering signal is developed in section (IV). The pneumatic system used for this experiment is developed in section (V), and it is tested by the new algorithm in section (VI). The results are compared to those obtained from the Neural Network.

Table 1. Nomenclature

\begin{tabular}{|c|l|}
\hline${ }^{-1}, T$ & $\begin{array}{l}\text { Notations denoting an inverse and matrix transpose, } \\
\text { respectively. }\end{array}$ \\
\hline||$_{\text {ABS }}$ & Absolute value. \\
\hline
\end{tabular}




\begin{tabular}{|c|c|}
\hline$\wedge$ & Estimation value. \\
\hline$A^{\circ} B$ & Schur product between $\mathrm{A}$ and $\mathrm{B}$. \\
\hline A & The system matrix. \\
\hline $\mathbf{B}$ & The input matrix. \\
\hline Ch & The chattering vector at time $\mathrm{k}$. \\
\hline d & The uncertainties vector of the SVSF. \\
\hline$\Delta$ & Difference between actual and estimated values. \\
\hline e & The .'s estimation error vectors. \\
\hline$E()$. & The expectation operator. \\
\hline$\gamma$ & The SVSF's positive constant matrix. \\
\hline H & The output matrix. \\
\hline $\mathbf{I}_{n \times n}$ & The identity matrix with dimensions of $\mathrm{n} \times \mathrm{n}$. \\
\hline$k$ & Time step value. \\
\hline$k \mid k$ & The a posteriori value at time $\mathrm{k}$. \\
\hline$k \mid k-1$ & The a priori value at time $\mathrm{k}$. \\
\hline $\mathbf{K}_{S V S F}$ & $\begin{array}{l}\text { The correction gain of the Smooth Variable Structure } \\
\text { Filter. }\end{array}$ \\
\hline $\lim _{b \rightarrow c} a$ & The value of a when $\mathrm{b}$ approaches $\mathrm{c}$. \\
\hline$\Psi$ & The smoothing boundary layer vector. \\
\hline RMSE & The root mean square error. \\
\hline$\sum_{i=b}^{c} \mathbf{a}_{i}$ & The summation of vector a from time $b$ to time $c$. \\
\hline $\operatorname{sgn}(a)$ & The sign function of the vector a. \\
\hline $\operatorname{sgn}(a)$ & The sign function of the element a. \\
\hline$u$ & The input. \\
\hline $\mathbf{x}$ & The state vector. \\
\hline $\mathbf{z}$ & The output vector. \\
\hline$a, b$ & Subscripts for inlet and outlet chambers, respectively \\
\hline$A$ & Ram area \\
\hline $\begin{array}{c}P_{e}, P_{d}, \\
P_{a t m}, P_{s}, \\
P_{u}, P_{d r o p}\end{array}$ & $\begin{array}{l}\text { Exhaust, Down-stream, Atmosphere, Supply, Upstream } \\
\text { and drop pressures, respectively. }\end{array}$ \\
\hline$L$ & Half the stroke length \\
\hline V & Volume \\
\hline$C_{d}, C_{f}$ & $\begin{array}{l}\text { Discharge and Viscous damping coefficients, } \\
\text { respectively. }\end{array}$ \\
\hline$F_{c}, F_{\text {static }}$ & Coulomb and Static frictions, respectively. \\
\hline$\dot{m}$ & Mass flow rate \\
\hline$M$ & Payload \\
\hline$P_{r}$ & Downstream to upstream pressure ratio \\
\hline$r$ & Specific heat constant \\
\hline$R$ & Gas constant \\
\hline$T_{s}, T_{u}$ & Supply and Upstream temperatures, respectively. \\
\hline$W$ & Port width \\
\hline$X$ & Spool displacement of valve \\
\hline$Y$ & Load position \\
\hline$\lambda_{L}$ & Material friction factor \\
\hline$\rho$ & Air density \\
\hline$L_{p}$ & Pipe's length \\
\hline$v$ & Average air velocity \\
\hline$D$ & Pipe's inner diameter \\
\hline
\end{tabular}

II. The Smooth Variable Structure Filter

In 2007, a revised version of the Variable Structure Filter, referred to as the Smooth Variable Structure Filter (SVSF), was proposed, [1]. The SVSF is a predictor corrector filter that is based on the SMC principles and can be applied to both linear and nonlinear systems. A requirement of this filter is that the system is differentiable and hence the word "smooth" is used to name this filter. The filter also requires that the system under consideration be observable, [1]

The SVSF's derivation depends on the rank of the measurement matrix. If the measurement matrix has partial rank, the SVSF's gain is calculated by using Luenberger's reduced order technique. This paper considers the case of a system that has full rank measurement matrices. For these systems, the SVSF process can be summarized in Figure 1.

\begin{tabular}{|c|c|c|}
\hline $\begin{array}{c}\text { Prediction } \\
\text { Stage }\end{array}$ & $k=0, \widehat{x}_{0 \mid 0}$ & $u_{k-1}$ \\
\hline$k=k+1$ & $\begin{array}{r}\hat{\mathbf{x}}_{k \mid k-1}= \\
\mathbf{e}_{\mathbf{z}_{k \mid}}\end{array}$ & $\begin{array}{l}-1+\widehat{\mathbf{B}}_{k-1} u_{k-1} \\
\widehat{\mathbf{I}}_{k} \widehat{\mathbf{x}}_{k \mid k-1}\end{array}$ \\
\hline $\begin{array}{r}U p d a \\
\mathbf{K}_{s_{k}}=\mathbf{H} \\
\hat{\mathbf{x}}_{k}\end{array}$ & $\begin{array}{l}\frac{\operatorname{tage}}{\left(\boldsymbol{\gamma} \mid \mathbf{e}_{\mathbf{z}_{k-1 \mid k-1}}\right.} \\
\hat{\mathbf{x}}_{k \mid k-1}+\mathbf{K}_{l}\end{array}$ & $\begin{array}{l}{ }^{\circ} \mathbf{s g n}\left(\mathbf{e}_{\mathbf{z}_{k \mid k-1}}\right) \\
-\widehat{\mathbf{H}}_{k} \widehat{\mathbf{x}}_{k \mid k}\end{array}$ \\
\hline
\end{tabular}

Figure 1: The Smooth Variable Structure Filter Steps [1].

The SVSF defines a hyperplane, which is referred to as the sliding surface, and applies a discontinuous force on the estimate to make it reach the hyperplane in two stages; prediction and update stages. The estimates in each stage remain within subspace surrounding the sliding hyperplane, referred to as the Existence Subspace as illustrated in Figure 2. During each stage, an artificial noise referred to as Chattering is created depending on uncertainties and the SVSF coefficient matrix $\boldsymbol{\gamma}$. Chattering obtained in prediction stage is referred to as the secondary indicator of performance [1]. Conversely, chattering in the a posteriori estimate decays with time by the factor $\gamma^{k}$, therefore no information could be obtained [19].

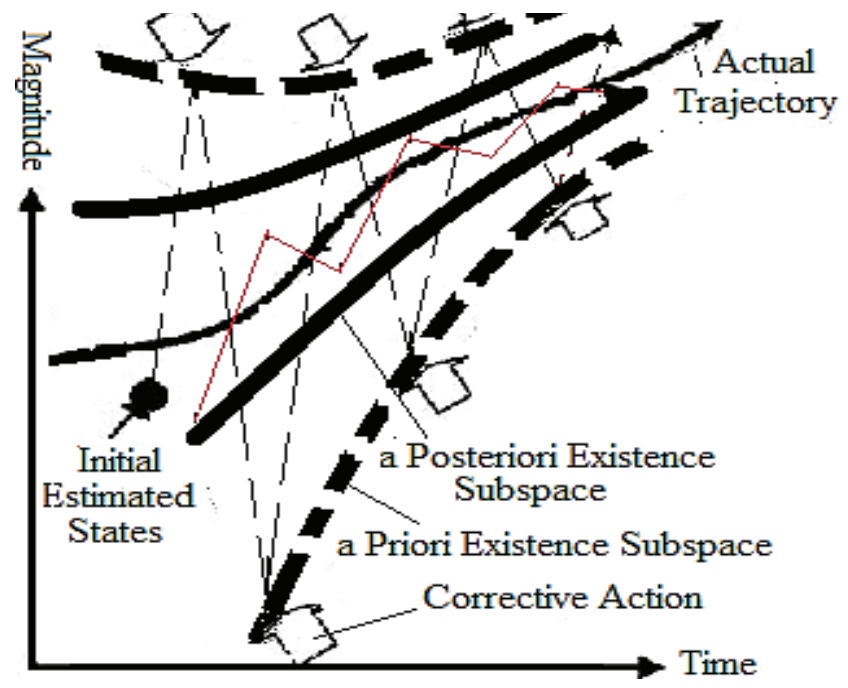

Figure 2: The SVSF Concepts [19], where — and - - are the a posteriori and the a priori estimated trajectories, respectively.

The estimate has two levels of chattering; the a priori and the a posteriori chattering. In order to eliminate these signals, the gain's sign function in Figure 1 is replaced by a smoothing function with a known boundary layer referred to as the smoothing boundary layer as in (1). Inside the smoothing boundary layer, the corrective action is interpolated based on the ratio between the amplitude of the output's a priori estimation error and the smoothing boundary layer's width. Outside the smoothing boundary layer, the discontinuous corrective action with its full amplitude is applied. The SVSF assigns and requires one smoothing boundary layer per estimate. 
$\mathbf{K}_{S V S F_{k}}=\widehat{\mathbf{H}}_{k}^{-1}\left(\boldsymbol{\gamma}\left|\mathbf{e}_{\mathbf{z}_{k-1 \mid k-1}}\right|+\left|\mathbf{e}_{\mathbf{z}_{k \mid k-1}}\right|\right){ }^{\circ} \mathbf{s a t}\left(\mathbf{e}_{\mathbf{z}_{k \mid k-1}}, \mathbf{\Psi}_{k}\right)$

$\boldsymbol{\Psi}_{k}$ must be chosen carefully, as large width causes a slower convergence rate and degrades filter performance, and smaller width causes chattering. If the width of the smoothing boundary layer is chosen to be larger than the width of the a priori existence subspace and the difference between them is made to be small, then chattering is removed and the error in the output estimation is limited. The width of the smoothing boundary layer determines the presence and the level of the a priori chattering. If the smoothing boundary layer is overestimated then chattering is removed. However, if due to changes in the system, additional uncertainties are added such that the amplitude of the output's a priori estimation error grows larger than the width of the smoothing boundary layer, then chattering will be observed, $[1,19]$. This gives the SVSF the ability to explicitly point out and extract information on modeling uncertainties. Therefore, chattering is considered as the secondary indicators of performance.

In this section, equations are derived to quantify the amplitudes of the a priori and the a posteriori chattering.

\section{A. The a posteriori chattering}

The a posteriori chattering is derived in this section. Subtracting the actual measurement from the a posteriori estimated measurement yields:

$\mathbf{e}_{\mathbf{z}_{k \mid k}}=\mathbf{e}_{\mathbf{z}_{k \mid k-1}}-\mathbf{H}_{k} \mathbf{K}_{S V S F_{k}}$

(2) could be simplified to:

$\mathbf{e}_{\mathbf{z}_{k \mid k}}=-\boldsymbol{\gamma}\left|\mathbf{e}_{\mathbf{z}_{k-1 \mid k-1}}\right|{ }^{\circ} \operatorname{sgn}\left(\mathbf{e}_{\mathbf{z}_{k \mid k-1}}\right)$

For the SVSF with zero width-smoothing boundary layer, the a posteriori chattering is then defined as:

$\mathbf{C h}_{k \mid k}=-\boldsymbol{\gamma}^{k}\left|\mathbf{e}_{\mathbf{z}_{0 \mid 0}}\right|{ }^{\circ} \operatorname{sgn}\left(\mathbf{e}_{\mathrm{z}_{k \mid k-1}}\right)$

Knowing that $\boldsymbol{\gamma}$ is a diagonal matrix that has elements with magnitudes less than unity, leads the chattering to decay with time.

\section{B. The a priori chattering signal}

The a priori estimation error is defined as follows:

$\mathbf{e}_{\mathbf{z}_{k \mid k-1}}=\mathbf{z}_{k}-\widehat{\mathbf{z}}_{k \mid k-1}=\mathbf{H}_{k} \mathbf{x}_{k}+\mathbf{v}_{k}-\widehat{\mathbf{H}}_{k} \hat{\mathbf{x}}_{k \mid k-1}$

Substituting system equations of Figure 1 into (5) gives:

$\mathbf{e}_{\mathbf{z}_{k \mid k-1}}=\left[\begin{array}{c}\mathbf{H}_{k}\left(\mathbf{A}_{k-1} \mathbf{x}_{k-1}+\mathbf{B}_{k-1} u_{k-1}+\mathbf{w}_{k-1}\right) \\ -\widehat{\mathbf{H}}_{k}\left(\widehat{\mathbf{A}}_{k-1} \widehat{\mathbf{x}}_{k-1 \mid k-1}+\widehat{\mathbf{B}}_{k-1} u_{k-1}\right)\end{array}\right]+\mathbf{v}_{k}$

Assuming the output matrix is the identity matrix, using the measurement equation, and rearranging (6) give:

$\mathbf{e}_{\mathbf{z}_{k \mid k-1}}=\left[\begin{array}{c}\left(\mathbf{A}_{k-1} \mathbf{z}_{k-1}-\mathbf{A}_{k-1} \mathbf{v}_{k-1}+\mathbf{B}_{k-1} u_{k-1}\right) \\ -\left(\widehat{\mathbf{A}}_{k-1} \hat{\mathbf{z}}_{k-1 \mid k-1}+\widehat{\mathbf{B}}_{k-1} u_{k-1}\right)+\mathbf{v}_{k}+\mathbf{w}_{k-1}\end{array}\right]$

The a posteriori estimated measurement is defined as [19]:

$\hat{\mathbf{z}}_{k-1 \mid k-1}=\mathbf{z}_{k-1}+\boldsymbol{\gamma}\left|\mathbf{e}_{\mathbf{z}_{k-2 \mid k-2}}\right|{ }^{\circ} \mathbf{s g n}\left(\mathbf{e}_{\mathbf{z}_{k-1 \mid k-2}}\right)$

Substituting (8) and (3) into (7), rearranging and assuming time invariant system yield:

$\mathbf{e}_{\mathbf{z}_{k \mid k-1}}=\left[\begin{array}{c}\Delta \mathbf{A} \mathbf{z}_{k-1}+\Delta \mathbf{B} u_{k-1}+\mathbf{w}_{k-1}+\mathbf{v}_{k} \\ -\mathbf{A} \mathbf{v}_{k-1}-\widehat{\mathbf{A}} \boldsymbol{\gamma}^{k-1}\left|\mathbf{e}_{\mathbf{z}_{0 \mid 0}}\right|{ }^{\circ} \operatorname{sgn}\left(\mathbf{e}_{\mathbf{z}_{k-1 \mid k-2}}\right)\end{array}\right]$

From (9), the terms $(\Delta \mathbf{A})$ and $(\Delta \mathbf{B})$ capture the influence of the modeling errors. The terms $\left(\mathbf{w}_{k-1}+\mathbf{v}_{k}-\mathbf{A} \mathbf{v}_{k-1}\right)$ quantifies the impact of the system and measurement noise. The last term in (9), describes the effects of the uncertainty in initial conditions. According to the latter term, the effect of the error in initial conditions decays in time at a rate of $\gamma^{\boldsymbol{k}-\mathbf{1}}$, and becomes negligible as $k \rightarrow \infty$.
The a priori existence subspace represents the error in the a priori estimate. In other words, it describes the chattering of the a priori estimate around the true trajectory. In this paper, the magnitude of the resultant chattering is referred to as the $\boldsymbol{a}$ priori chattering. Because of the predictor-corrector nature of the SVSF and its gain, the a priori chattering is different from the chattering observed in other SMOs [19]. The a priori chattering is then obtained by eliminating the effect of initial conditions as:

$\mathbf{C h}_{k \mid k-1}=\Delta \mathbf{A z}_{k-1}+\Delta \mathbf{B} u_{k-1}+\mathbf{w}_{k-1}+\mathbf{v}_{k}-\mathbf{A v}_{k-1}$

\section{THE CHATTERING AMPLITUDES AND ITS INFORMATION}

\section{CONTENT}

The a priori chattering can be used to point out the source and amplitude of modeling errors. Chattering gives an indication that the current filter's model is uncertain and it needs to be re-estimated or tuned. Chattering thus provides an opportunity for combining the SVSF with adaptive techniques for model refinement.

According to (12), the SVSF has $n$-a priori chattering signals, one associated with each estimate (assuming that there is $n$-states). Thus, each chattering signal points out the modeling errors in the corresponding row of the system and input matrices. As such, the a priori chattering contents provide means for extracting the modeling error explicitly. In this paper, an approach is presented to obtain these uncertainties assuming the input is non-stationary. In order for this method to work, the measurement and system noise signals must be stationary. Since the expectation vector is not directly available, a segment of the a priori chattering is taken and its statistics are calculated; i.e. mean. Using the law of large numbers, the means vectors of the measurement and system noise in the a priori chattering segment approach their expectations and hence they are known.

\section{Lemma - The Law of Large Numbers [6]}

The Law of Large Numbers states that: if $d$ stationary uncorrelated random variables, such as $v_{i}, i=1 \ldots d$, share the same expectation value, then their average tends towards its expected value as $d \rightarrow \infty$ as follows, [6]:

If $E\left[v_{1}\right]=E\left[v_{2}\right]=\cdots=E\left[v_{d}\right]=\bar{v}$ then $\lim _{d \rightarrow \infty}\left(\frac{1}{d} \sum_{i=1}^{d} v_{i}\right)=\bar{v}$.

The method involves obtaining the modeling errors of a time invariant system from the a priori chattering signals by using an averaging technique. This can be done by taking a segment of length $d$ (starting from the time step $l$, when the term $\boldsymbol{\gamma}^{l}$ becomes negligible, to time step $l+d-1$ ) from the a priori chattering, the input and the measurement signals, and then calculating their averages. From (12), the average of the chattering segment is obtained as:

$\frac{1}{d} \sum_{i=l}^{l+d-1} \mathbf{C h}_{i \mid i-1}=\frac{1}{d}\left[\begin{array}{c}\Delta \mathbf{A} \sum_{i=l}^{l+d-1} \mathbf{z}_{i-1}+\Delta \mathbf{B} \sum_{i=l}^{l+d-1} u_{i-1} \\ +\sum_{i=l}^{l+d-1}\left(\mathbf{w}_{i-1}+\mathbf{v}_{i}-\mathbf{A v}_{i-1}\right)\end{array}\right]$

If $d$ is chosen to be large enough to satisfy the lemma, then the averages of the measurement and system noise converge to their expectations which are zero (zero-mean assumption) as:

$\lim _{d \rightarrow \infty}\left(\frac{1}{d} \sum_{i=l}^{l+d-1}\left(\mathbf{w}_{i-1}+\mathbf{v}_{i}-\mathbf{A} \mathbf{v}_{i-1}\right)\right)=\mathbf{0}_{n \times 1}$

If the measurement matrix is the identity matrix, system and measurement noise are white, and the smoothing boundary layer is set to have zero width, then (13) is simplified to: 
$\lim _{d \rightarrow \infty}\left(\frac{1}{d} \sum_{i=l}^{l+d-1} \mathbf{C h}_{i \mid i-1}\right)=\lim _{d \rightarrow \infty}\left(\begin{array}{c}\frac{1}{d} \Delta \mathbf{A} \sum_{i=l}^{l+d-1} \mathbf{z}_{i-1} \\ +\frac{1}{d} \Delta \mathbf{B} \sum_{i=l}^{l+d-1} u_{i-1}\end{array}\right)$.

(15) has $(n+1) \times n$ unknowns which are the following:

- $n \times n$ modeling errors in the system matrix, $\Delta \mathbf{A}$.

- $\quad n \times 1$ modeling errors in the input matrix, $\Delta \mathbf{B}$.

To solve these unknowns, $(n+1) \times n$ equations are needed. Each segment provides $(n+1)$ data points that represent the average values of $n$-measurement and one-input in that segment. Therefore using $n$ segments provides $n$ data points of vector $\left[\begin{array}{l}\sum_{i=l}^{l+d-1} \mathbf{z}_{i-1} \\ \sum_{i=l}^{l+d-1} \mathbf{u}_{i-1}\end{array}\right]$, which gives $(n+1) \times n$ equations. This allows the problem to be solved. Using these vectors, (15) is then revised as follows:

$\lim _{d \rightarrow \infty}\left(\frac{1}{d}\left[\begin{array}{c}\sum_{i=l_{1}}^{l_{1}+d-1} \mathbf{C h}_{i \mid i-1} \\ \vdots \\ \sum_{i=l_{n}}^{l_{n}+d-1} \mathbf{C h}_{i \mid i-1}\end{array}\right]^{T}\right)=$

$\left[\begin{array}{ll}\Delta \mathbf{A} & \Delta \mathbf{B}\end{array}\right] \lim _{d \rightarrow \infty}\left(\frac{1}{d}\left[\begin{array}{lll}\sum_{i=l_{1}}^{l_{1}+d-1} \mathbf{z}_{i-1} & \cdots & \sum_{i=l_{n}}^{l_{n}+d-1} \mathbf{z}_{i-1} \\ \sum_{i=l_{1}}^{l_{1}+d-1} u_{i-1} & \cdots & \sum_{i=l_{n}}^{l_{n}+d-1} u_{i-1}\end{array}\right]\right)$

Where $l_{i}, i=1, \ldots, n$ represents the beginning of the $i^{t h}$ segment. (16) is expressed in a simpler form as:

$\mathbf{C H}=\left[\begin{array}{ll}\Delta \mathbf{A} & \Delta \mathbf{B}\end{array}\right]\left[\begin{array}{l}\overline{\mathbf{Z}} \\ \overline{\mathbf{U}}\end{array}\right]$

Note that the input must not be stationary. From (17) the modeling errors can be obtained as follows:

$\left[\begin{array}{ll}\Delta \mathbf{A} & \Delta \mathbf{B}\end{array}\right]=\mathbf{C H}\left[\frac{\overline{\mathbf{Z}}}{\overline{\mathbf{U}}}\right]^{-1}$

(18) can be used to adapt the filter's model. Due to the expectation operator, the extracted parameters may differ from their real values. If the refined model is close to the system model, then the a priori chattering due to modeling error becomes negligible. Therefore, the a priori chattering becomes a function of the measurement and system noise. If the refined model differs from the system model due to the expectation operator, then the output's a priori estimation error exceeds the smoothing boundary layer and thus a priori chattering appears and indicates that the filter model needs to be re-tuned. The refinement's procedure is then repeated. Due to the choice of the segment length and the usage of the expectation operator, the refinement procedure may be repeated several times. Once the filter is tuned, the SVSF's smoothing boundary layer width changes to be larger than the noise uncertainties. If modeling errors are present again, the smoothing boundary layer width is set to be zero in order to enable the refinement's process. After the refinement procedure is completed, the boundary layer is changed back to be larger than the noise uncertainties.

\section{Pneumatic System Mathematical Model}

This section presents the mathematical model of a pneumatic system that consists of double acting asymmetric cylinder, Five-port proportional valve, load, and tubes.

\section{A. Cylinder model}

Assuming that chamber $\mathrm{A}$ is the driving chamber $\left(P_{a}>P_{b}\right)$, then the control volumes in the cylinder chamber is:

$\dot{m}_{a} C_{p} T_{a v}-\dot{m}_{L} C_{p} T_{a}-P_{a} d V_{a} / d t=d\left(C_{v} \rho_{a} V_{a} T_{a}\right) / d t$

Similarly, if chamber B is the driving chamber $\left(P_{a}<P_{b}\right)$, then:

$\dot{m}_{a} C_{p} T_{b v}-\dot{m}_{L} C_{p} T_{b}-P_{a} d V_{b} / d t=d\left(C_{v} \rho_{a} V_{b} T_{b}\right) / d t$

Assuming an ideal gas $(P=R \rho T$ and $P / R=\rho T)$, then the right hand side can be simplified to:

$C_{v} \rho_{a} V_{a} T_{s}=C_{v} P_{a} V_{a} / R$
There are two unknowns $\left(P_{a}, P_{b}\right)$ in these equations. Rearrange for $P_{a}$ and $P_{b}$ :

$P_{a}=\int\left(\left(\dot{m}_{a}+\dot{m}_{L}\right) r R T_{a}-r A_{a} P_{a} \dot{Y}\right) /\left(A_{a} Y\right)$
$P_{a}=\int\left(\left(\dot{m}_{b}+\dot{m}_{L}\right) r R T_{b}-r A_{b} P_{b} \dot{Y}\right) /\left(A_{b}(L-Y)\right)$

\section{B. Load Model}

The dynamic behavior of the load is derived as:

$\left(A_{a} P_{a}-A_{b} P_{b}\right)-\left(A_{a}-A_{b}\right) P_{a t m}-F_{l}=F_{f} \operatorname{sign}(\dot{Y})+M \ddot{Y}+c_{f} \dot{Y} \quad$ (24) For the pneumatic cylinder considered in this paper, the friction model is simplified to obtain the more commonly used threshold model. (25) describes the adopted model.

$F_{f}=\left\{\begin{array}{cc}F_{c} & |\dot{Y}|>\delta v \\ F_{\text {static }} & |\dot{Y}| \leq \delta v\end{array}\right.$

C. Valve Model

According to the standard orifice theory, the mass flow rates across the two control ports of the control valve can be regarded as a function of the valve spool displacement and the chamber pressures, which can be expressed as

$\dot{m}_{a}=C_{d} C_{o} w X f_{p a}\left(P_{r}\right)$

$\dot{m}_{b}=-C_{d} C_{o} w X f_{p b}\left(P_{r}\right)$

For air $C_{d}=0.8, C_{o}$ can be expressed as following:

$C_{o}=\sqrt{r /\left(R\left(\frac{r+1}{2}\right)^{\frac{r+1}{r-1}}\right)}$

where $r=1.4, R=280$, then $C_{o}=0.04$.

The mass flow function for chamber $\mathrm{A}$ and chamber $\mathrm{B}$ can be expressed as

$f_{p a}\left(P_{r}\right)=\left\{\begin{array}{l}P_{s} / \sqrt{T_{s}} \tilde{f}\left(P_{a} / P_{s}\right) \quad \text { chamber } A \text { is a drive } \\ P_{a} / \sqrt{T_{a}} \tilde{f}\left(P_{e} / P_{a}\right) \quad \text { chamber } A \text { is an exhaust }\end{array}\right.$

and

$f_{p b}\left(P_{r}\right)=\left\{\begin{array}{l}P_{s} / \sqrt{T_{s}} \tilde{f}\left(P_{b} / P_{s}\right) \quad \text { chamber } B \text { is a drive } \\ P_{b} / \sqrt{T_{b}} \tilde{f}\left(P_{e} / P_{b}\right) \quad \text { chamber } B \text { is an exhaust }\end{array}\right.$

The above component-based model was created and simulated using MatLab/Simulink as shown in Figure 3.

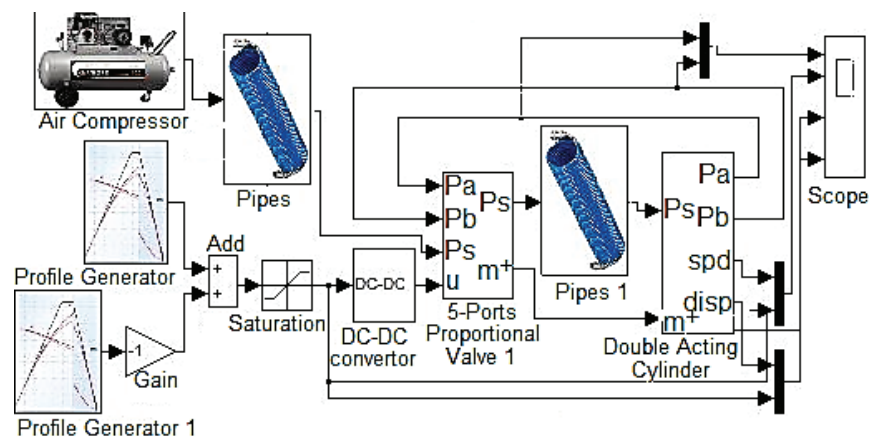

Figure 3: Simulink diagram of the simulated pneumatic system

V. THE APPLICATION OF THE SVSF INTO THE PNEUMATIC SYSTEM.

The SVSF's chattering signal can be used to tune the filter model in order to reduce the modeling errors. This was tested and verified by applying the SVSF to the pneumatic system described in the previous section. The basic idea of this experiment was to create a linearized system that gives the best approximation of the non-linear parts in the actual system. Using the chattering equation, the approximated linear system was tested every time step to ensure its efficiency. The results obtained from the SVSF are compared to the results obtained from a Neural Network with three hidden layers. 


\section{A. Simulation setup}

The SVSF is designed to have $\boldsymbol{\gamma}=\mathbf{0 . 2} \times \mathbf{I}_{n \times n}$ and $\boldsymbol{\Psi}=\mathbf{0 . 0} \times \mathbf{I}_{n \times 1}$. The order of the filter model is unknown as the filter model supposed to approximate the non-linear system model. Therefore, several iterations have been used with different order values to obtain the best value that reduces the root mean squared error. The uncertainties caused from the linearization process should have magnitudes less than $10 \%$ of the corresponding states' magnitudes. The filter uses a sample consisting of 200 points from the chattering, the measurements, and the input signals to extract the modeling errors (system parameters) as discussed in section (IV). The NN used is a feedforward network architecture with four layers: one input layer with six nodes, two hidden layers with 15 nodes each, and an output layer with three nodes. The six inputs used were the two random inputs (at time steps $\mathrm{k}$ and $\mathrm{k}-1$ ), two position signals (at time steps $\mathrm{k}$ and $\mathrm{k}-1$ ), one velocity signal (at time step k-1) and one acceleration signal (at time step k-1). The three outputs were the position, velocity, and acceleration (at time step k). The training algorithm used was the LevenbergMarquardt Backpropagation.

The experiment consisted of two parts: training (2297 patterns) and testing (10,000 patterns). For the former part, a random input signal that has maximum absolute value of 10 volts was used to excite the system. The outputs and the input were then used to obtain the system model or its equivalent by training the NN and the SVSF. The outputs are shown in Figures 4 to 6 . The model/neurons were then used in the latter part with different input profile to obtain the estimated states which are the position, velocity and acceleration. The tested input and outputs are shown in Figures 7 to 10.

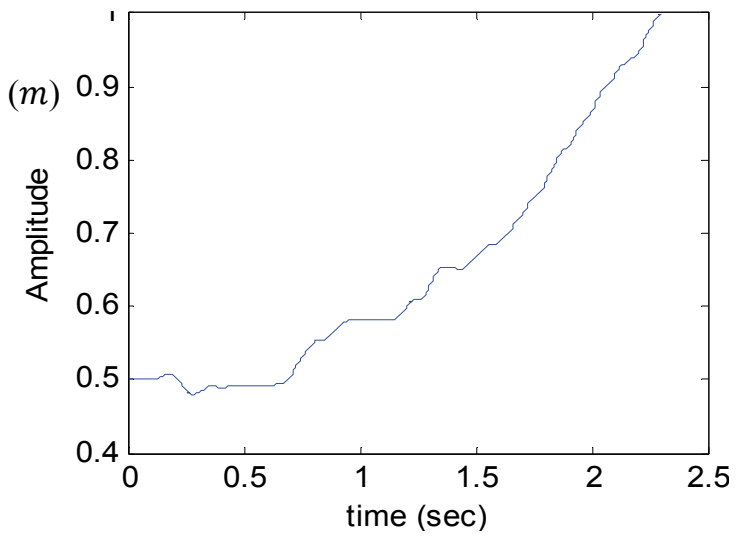

Figure 4: The training position for NN and SVSF

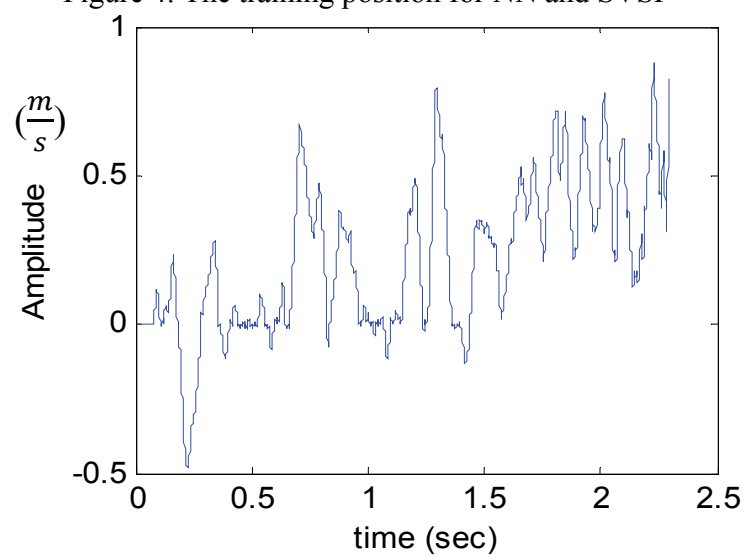

Figure 5: The training velocity for NN and SVSF

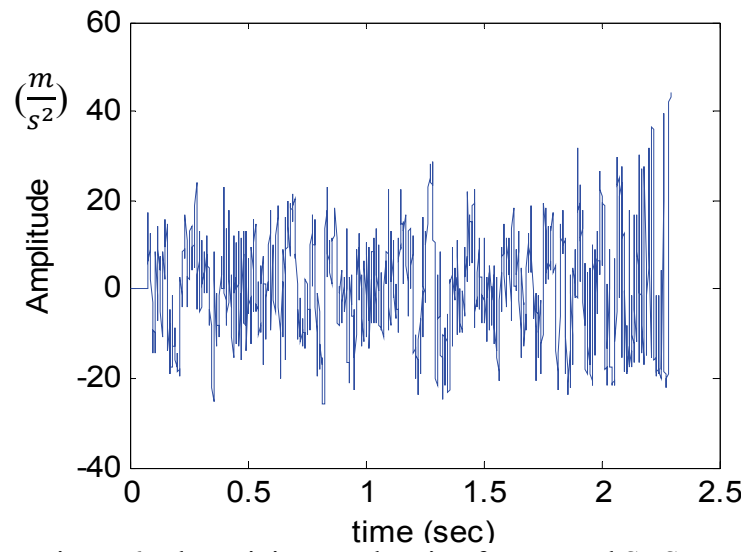

Figure 6: The training acceleration for NN and SVSF

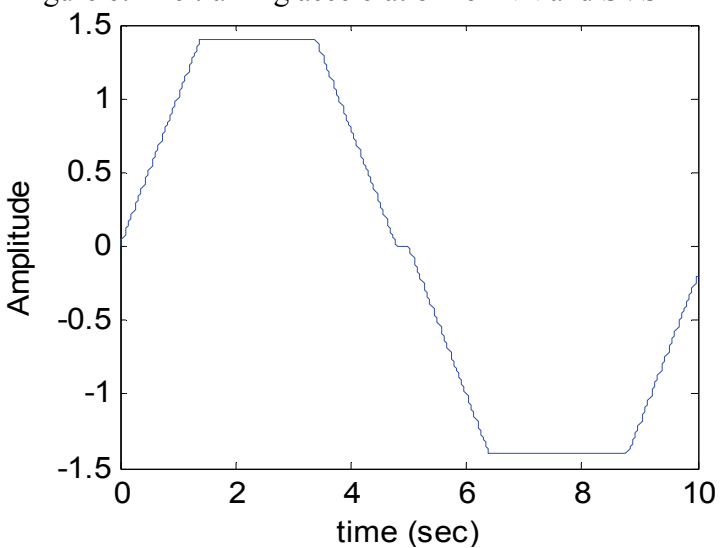

Figure 7: The input used in testing NN and SVSF

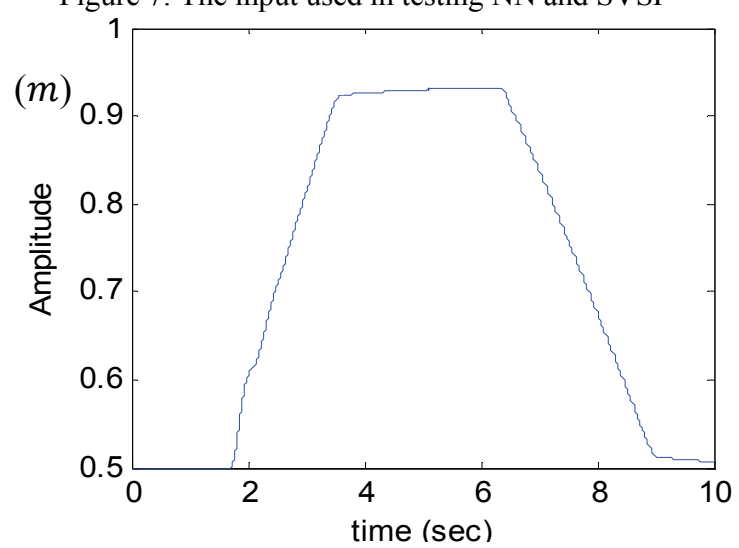

Figure 8: The position used in testing NN and SVSF

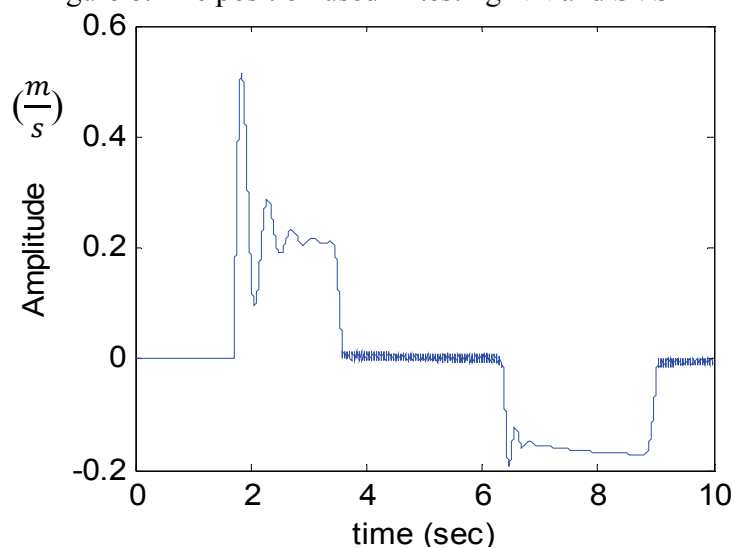

Figure 9: The velocity used in testing NN and SVSF 


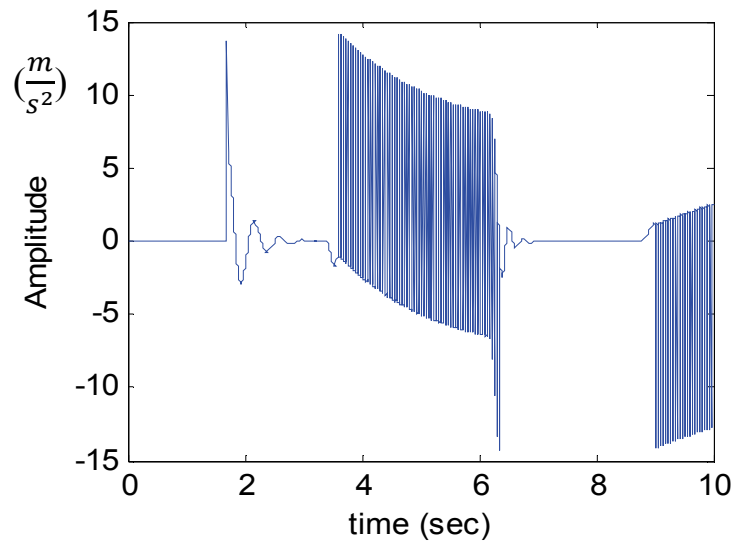

Figure 10: The acceleration used in testing NN and SVSF

\section{B. Simulation results}

Figure 11 shows the results of the proposed method compared to the results obtained from Neural Network. The results show that the SVSF gives good estimation for the states and the parameters of the system where the root mean squared errors (RMSE) are less than $10^{-20}$. The advantages of using the SVSF beside the high accuracy results are:
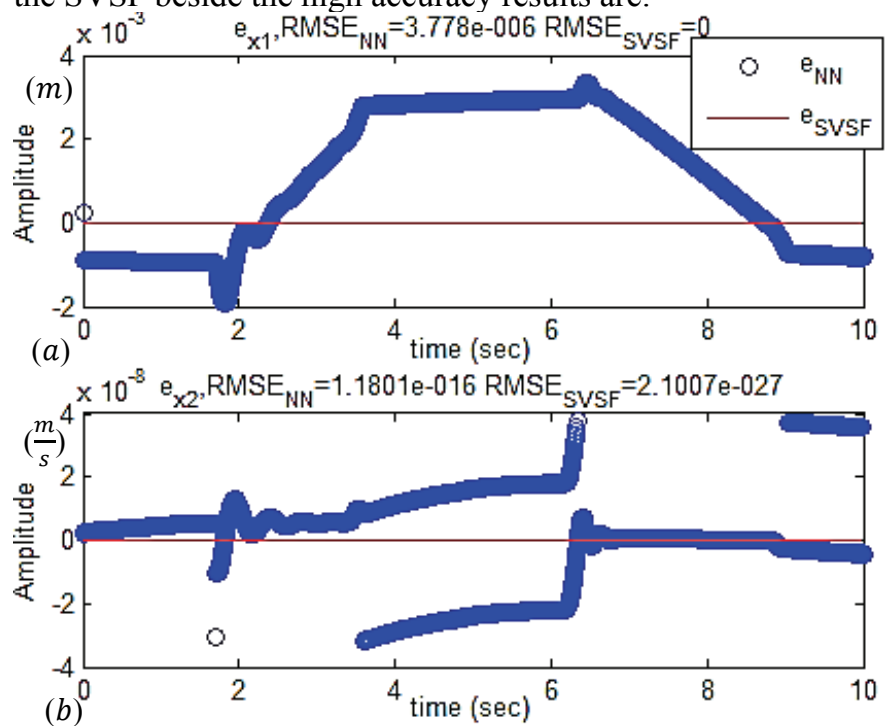

(b) $\quad \mathrm{e}_{\mathrm{x} 3}, \mathrm{RMSE}_{\mathrm{NN}}=37.2862 \mathrm{RMSE}_{\mathrm{SVSF}}=6.1186 \mathrm{e}-021$

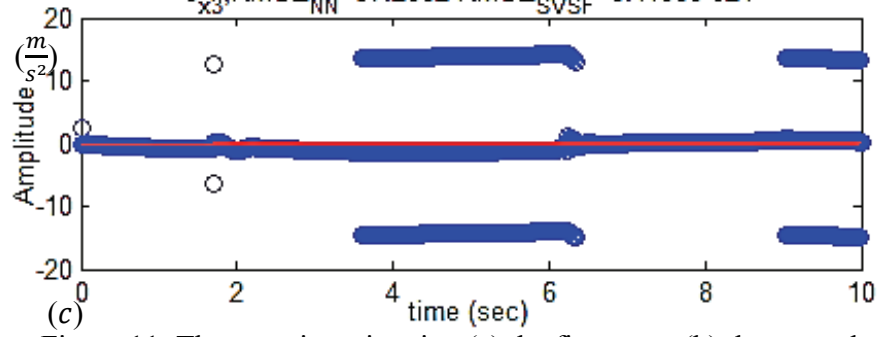

Figure 11: The error in estimating (a) the first state, (b) the second state and (c) the third state.

1- The speed of convergence. It took 2 iterations to obtain the parameters.

2- The easiness of tuning, and implementing the algorithm for online applications.

3- The ability of adapting any further changes in the system's parameters. This gives the proposed algorithm the flexibility needed in approximating non-linear systems by using several piece-wise linear subsystems.

\section{CONCLUSION}

This work considers the application of the SVSF to a pneumatic system application. The SVSF is a recently proposed predictor-corrector filter for state and parameter estimation, and it is based on the sliding mode control concepts. The characteristics of the smoothing boundary layer were briefly described. In this paper, a novel strategy was presented to obtain the system information (i.e. parameters) by using the SVSF's a priori chattering signal. A simple algebraic algorithm could be used to identify and extract the modeling uncertainties from the chattering signal. This method was tested on a pneumatic system and the results showed that the proposed method is stable and has performance that exceeds that of the Neural Network.

\section{REFERENCES}

[1] S. Habibi, "The Smooth Variable Structure Filter," Proceedings of the IEEE, 95(5), 1026 - 1059, 2007.

[2] T. Kailath, "A View Of Three Decades Of Linear Filtering Theory," IEEE Transactions on Information Theory, IT-20(Issue 2), 146-181, 1974.

[3] H. Sorenson, "Least-Squares Estimation: From Gauss To Kalman," IEEE Spectrum, 7(7), 63-68, 1970.

[4] Z.Chen, "Bayesian Filtering: From Kalman Filters To Particles And Beyond," 2003.

[5] D. Simon, "Optimal State Estimation: Kalman, H [infinity] And Nonlinear Approaches," Wiley-Interscience, c2006.

[6] T. Bar-Shalom, X. Li, \& T. Kirubarajan, "Estimation With Applications To Tracking And Navigation - Theory, Algorithm And Software," John Wiley \& Sons, Inc, 2001.

[7] Y. Ho \& R. Lee, "A Bayesian Approach To Problems In Stochastic Estimation And Control," IEEE Transactions on Automatic Control, 9(4), 333-339, 1964.

[8] M. Barakat, "Signal Detection And Estimation," Norwood :Artech House, 2005

[9] F. Van Der Heidan, R. Duin, D. de Ridder \& C. Tax, "Classification, Parameter Estimation And State Estimation - An Engineering Approach Using MATLAB," England: John Wiley \& Sons, Ltd, 2004.

[10] J. Slotine, J. Hedrick \& E. Misawa, "On Sliding Observers for Nonlinear Systems," Proceedings of the 1986 American Control Conference. (pp. 1794 - 1800). Seattle, WA, USA: IEEE, 1986.

[11] J. Slotine, J. Hedrick \& E. Misawa, "On Sliding Observers for Nonlinear Systems," Transactions of the ASME. Journal of Dynamic Systems, Measurement and Control, 109(3), 245-252, 1987.

[12] B. Walcott, M. Corless \& S. Zak, "Comparative Study of State Observation Techniques," International Journal of Control, 45(6), 2109-2132, 1987.

[13] C. Edwards \& S. Spurgeon, "On the Development of Discontinuous Observers," International Journal of Control, 59, 1211 - 1229, 1994.

[14] C. Edwards, S. Spurgeon \& R. Patton, "Sliding Mode Observers for Fault Detections," Automatica, 36, 541 - 553, 2000.

[15] C. Tan \& C. Edwards, "Sliding Mode Observers for Robust Detection and Reconstruction of Actuator and Sensor Faults," International Journal of Robust and Nonlinear Control, 13, 443 - 463, 2003.

[16] S. Habibi \& R. Burton, "The Variable Structure Filter," 2002 ASME International Mechanical Engineering Congress and Exposition. 9, pp. 157 - 165. New Orleans, LA, United states: American Society of Mechanical Engineers, 2002.

[17] S. Habibi \& R. Burton, "The Variable structure Filter," Journal of Dynamic Systems, Measurement, and Control, 125, 287 - 293, 2003.

[18] S. Habibi \& R. Burton, "Parameter identification for a high performance hydrostatic actuation system using the variable structure filter concept," Proceedings of the ASME Fluid Power Systems and Technology Division. 11, pp. 93 - 101. Anaheim, CA, United states: American Society of Mechanical Engineers, 2004.

[19] M. Al-Shabi, "The General Toeplitz/Observability Smooth Variable Structure," McMaster University, Canada, PhD thesis, 2011.

[20] A. Saleem, "Component-based mixed reality environment for the control and design of servo pneumatic system," $\mathrm{PhD}$ thesis, De Montfort University, UK, 2006. 